\title{
Universidades de investigación: una paradoja emergente en la formación docente*
}

Research universities: an emerging paradox in teacher education

Citar como: Padilla Beltrán, J. E., Silva Carreño, W. H. y Colorado Ordoñez, P. (2020). Universidades de investigación: una paradoja emergente en la formación docente. CITAS, 6(1). https://doi.org/10.15332/24224529.6357

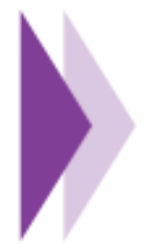

José Eduardo Padilla Beltrán ${ }^{1}$; Wilmer Hernando Silva Carreño ${ }^{2}$; Paula Colorado Ordoñez ${ }^{3}$

1 Universidad Militar Nueva Granada

2 Universidad de San Buenaventura

3 Universidad Militar Nueva Granada

Correo electrónico: edopadilla@gmail.comwisilca@gmail.com paula.colorado@unimilitar.edu.co 


\section{Resumen}

Este artículo caracteriza el sentido y alcance de una universidad de investigación y sus implicaciones en el papel docente: la formación en investigación, el uso de las herramientas electrónicas y la aplicación en la orientación de los aprendizajes. Se acudió al paradigma interpretativo comprensivo, con enfoque cualitativo, y se afincó en un estudio de caso entre los docentes de la Universidad Militar Nueva Granada. De esta caracterización emerge la importancia de una generación de académicos idóneos, que reclama una innovación y una apertura en sus enfoques y prácticas, aspectos fundamentales en la orientación de los aprendizajes de los estudiantes con el uso de herramientas electrónicas. Se concluye que la formación en investigación del docente, la idoneidad y el uso de tecnologías de la información y la comunicación, y de las tecnologías del aprendizaje, así como el conocimiento en su trabajo cotidiano, generan competencias que fácilmente se transmiten a su educando a través de ambientes virtuales de aprendizaje.

Palabras clave: investigación, innovación, universidades de investigación, rol docente, ambientes virtuales de aprendizaje.

Bien sea por un interés institucional o por exigencias externas gubernamentales y mercantiles, muchas universidades han adoptado el concepto y el compromiso de vincular la academia y la investigación como un binomio clave para el avance de la ciencia y como bases de la educación superior. En Colombia, una mirada somera al contexto universitario permite concluir que las universidades de investigación, es decir, aquellas que pueden articular con éxito tal binomio, representan solo una pequeña proporción en la educación superior y, sin embargo, su influencia es mayor que aquellas que no logran hacerlo.

Las universidades de investigación, a la vez que educan a estudiantes universitarios, capacitan a los profesionales y ofrecen servicios a la sociedad; además, pueden involucrarse en el trabajo aplicado y en la transferencia de tecnología haciendo de esto su característica distintiva. Es decir, en la producción de nuevos conocimientos en áreas de ciencia y tecnología se les destaca como instituciones clave para el desarrollo social y económico. Empero, para lograr esto, las universidades de investigación deben proporcionar la infraestructura académica, humana y física necesarias para llevar a cabo prácticas académicas e investigativas de alto nivel, de acuerdo con el Decreto n. ${ }^{\circ} 1330$ de 2019. A esto se suma que estas universidades se ven obligadas a mirar más allá de los límites de los países en los que están ubicadas para definir su alcance institucional, configurándose una naturaleza transnacional, en parte porque el espectro de competitividad se extiende globalmente.

Por otra parte, la formación del docente en cuanto a pedagogía, didáctica y evaluación de los aprendizajes hoy día se complementa con habilidades en el manejo de herramientas en tecnologías de información y comunicación (TIC), tecnologías de aprendizaje y conocimiento (TAC), tecnologías de emprendimiento y participación (TEP) y tecnologías emergentes (TE), que apoyan la orientación de los aprendizajes principalmente cuando se ofrece mediante ambiente virtuales de aprendizaje (González, Padilla y Montoya, 2019).

El objetivo de la investigación se fundamenta en la 'caracterización de la investigación electrónica de los docentes, su apropiación en la fundamentación en los saberes orientados a sus educandos a través de TIC, TAC, TEP y TE'. Por lo que se

\section{Abstract}

This article characterizes the meaning and scope of a research university and its implications in the teaching role: research training, use of electronic tools and application in the orientation of learning. The comprehensive interpretive paradigm was used, with a qualitative approach, and was based on a case study among teachers at the Universidad Militar Nueva Granada. From this characterization emerges the importance of a generation of qualified academics, who demand innovation, openness in their approaches and practices; fundamental aspects in the orientation of students' learning with the use of electronic tools. It is concluded that the teacher's education in research, the suitability and the use of information and communication technologies, and learning technologies, as well as the knowledge in their daily work, generates competencies that are easily transmitted to their students through virtual learning environments.

Keywords: research, innovation, research universities, teaching role, virtual learning environments.

indagó en cuanto a: ¿qué opinan quienes desarrollan o están a la base de toda práctica investigativa en las universidades? ¿Qué alcance y sentido tiene la innovación en la práctica investigativa de los docentes universitarios? ¿Qué tipos de herramientas usan para la apropiación del saber y la orientación del aprendizaje de sus estudiantes en el aula virtual? ¿Cuál es el papel de las redes de conocimiento en la apropiación del aprendizaje de los estudiantes?

\section{La formación y la práctica investigativa en la formación docente}

La formación investigativa en los docentes acompaña, de una parte, el desarrollo de habilidades para el estudio de un problema o fenómeno concreto, con la rigurosidad y sistematicidad que reclama el conocimiento de carácter científico; de otra, la consolidación de una formación profesional docente que contribuya a la búsqueda de solución a los problemas propios de un contexto social (Giroux, 2016).

La formación investigativa del docente, más allá de la práctica pedagógica a la cual se vea abocada, reivindica el papel de la pedagogía desde una perspectiva interdisciplinaria del saber, en tanto que enseñar no deviene como un saber constituido o prefigurado, sino que requiere hacerse continuamente; es decir, implica una actitud de búsqueda e indagación, y esta actitud investigativa posibilita el pensar y actuar científico e innovador. Sobre este punto señala Freire (1997), citado por Araújo (2017): se requiere es que el profesor, en su formación permanente, se perciba y se ocupe, por ser profesor, como investigador.

De este modo, docencia e investigación son un binomio de especial importancia para la práctica docente, pues el proceso de aprendizaje disciplinar en un estudiante tendrá mayor sentido por medio de la investigación, y mayor impacto en sus contextos si se les facilita la creación y gestión de procesos de investigación de las situaciones problemáticas que les afectan (Giroux, 2004).

Las instituciones de educación superior cuentan en su planta con profesionales en diferentes disciplinas y campos del conocimiento, pero la labor docente requiere de conocimientos en ciencias de la educación y altos fundamentos en pedagogía, didáctica, evaluación del aprendizaje y, en el caso de la educación 
virtual o a distancia, fundamentalmente habilidades en el uso de herramientas como las TIC, TAC, TEP y, entre otras, TE (González, Padilla y Montoya, 2019).

\section{Diseño metodológico}

Este artículo es derivado del proyecto de investigación Formación pedagógica e investigativa en tecnologías del aprendizaje $y$ conocimiento para las licenciaturas en proceso de acreditación, código INV DIS 2321, avalado y financiado por la Universidad Militar Nueva Granada en la vigencia 2017. Con este proyecto se analizó la implementación de la investigación electrónica por los docentes de la Universidad Militar Nueva Granada (UMNG) y su impacto en la producción del conocimiento. Para el desarrollo del proyecto, de corte cualitativo, se siguió el paradigma interpretativo\#comprensivo con recurso al estudio de caso como estrategia metodológica, y al análisis e interpretación de entrevistas a profundidad como instrumento de investigación.

La población informante se constituyó con docentes investigadores de la UMNG, clasificados en Colciencias en las categorías júnior, asociado y sénior en la convocatoria para el Reconocimiento de Investigadores del Sistema Nacional de Ciencia, Tecnología e Innovación (SNCTeI) 2015. Se entrevistaron a quince profesores en total: ocho con la categoría de investigador júnior, seis con la categoría de investigador asociado y uno con la categoría de investigador sénior.

En el desarrollo de las entrevistas se asumieron siete preguntas generadoras de información. En este artículo se analizan las respuestas y la información brindada por los profesores entrevistados a dos de estas preguntas, las cuales fueron: 1) En su opinión, ¿cuáles serían las competencias o habilidades de un excelente investigador? Nombre solo cuatro, siendo la primera la de mayor importancia; y 2) En cuanto al futuro de la investigación, ¿cuál es su apreciación respecto a la innovación en la formación investigativa?

Por el carácter de la estrategia metodológica (estudio de caso) y del instrumento (entrevista a profundidad), este último se construyó a partir del fundamento teórico y de discusiones grupales, con el fin de indagar sistemáticamente sobre los aspectos relacionados con la investigación a partir de la experiencia personal, del proceso de formación académica y de la labor y desempeño profesional de los informantes. En general, el proceso preliminar, sistemático e interpretativo de la información pasó por diversas fases, a saber: 1) establecimiento de las categorías de estudio en una matriz de análisis, tomando como sedimento una fundamentación conceptual; 2) una vez hecha la categorización, se procedió a estructurar la encuesta de preguntas abiertas, el instrumento contó con su respectiva validez de contenido, lo que permitió decantar algunas preguntas y diseñar la encuesta definitiva; 3 ) posterior a la aplicación de la encuesta, la información recopilada se llevó a una tabla de registro, donde se estructuraron o se conformaron las diferentes categorías ya existentes y las emergentes; y 4) se analizaron los resultados en tres etapas, la primera hace referencia al análisis de las respuestas de los encuestados, la segunda al cuadro comparativo de los resultados de las encuestas y las definiciones de las categorías desde el planteamiento del problema, y la tercera etapa se refiere al análisis interpretativo de lo reportado por los docentes.

\section{Resultados}

En la figura 1 se muestran las caracterizaciones que tienen los profesores entrevistados en relación con las cualidades de un investigador en la UMNG. 
Figura 1. Caracterización de los profesores en torno a las cualidades de un investigador en la UMNG

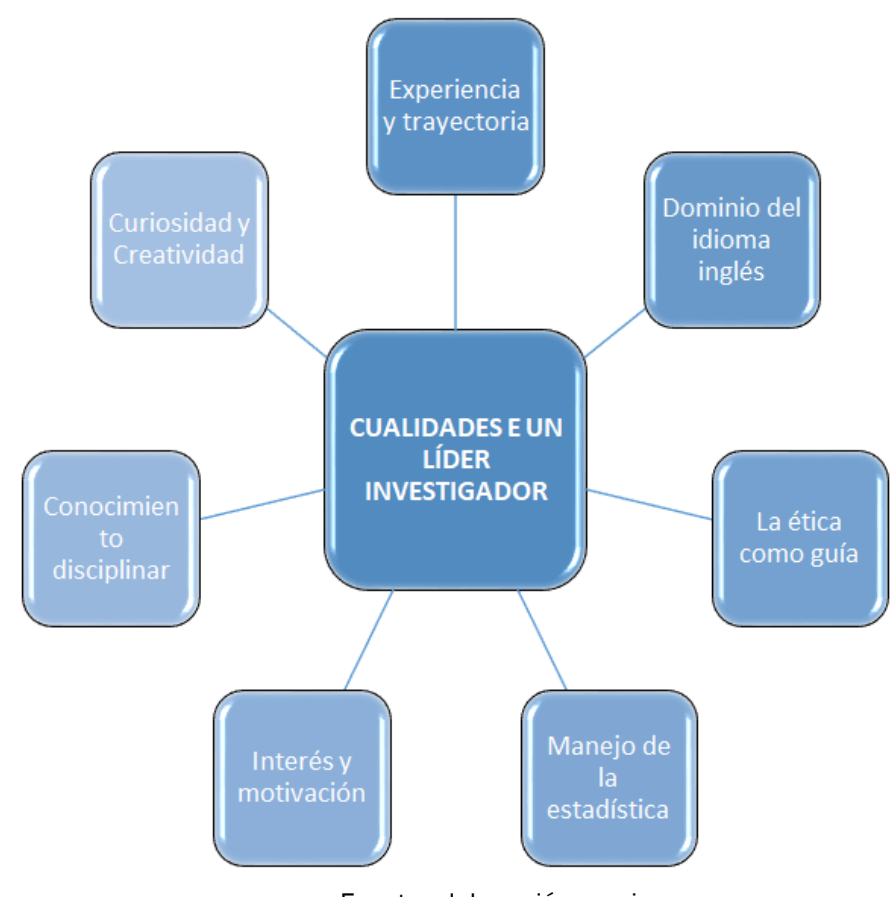

Fuente: elaboración propia.

En la figura 2 se muestran las caracterizaciones que tienen los profesores entrevistados en relación con las implicaciones de la innovación investigativa en la UMNG. 
Figura 2. Caracterización de los profesores en torno a la innovación investigativa

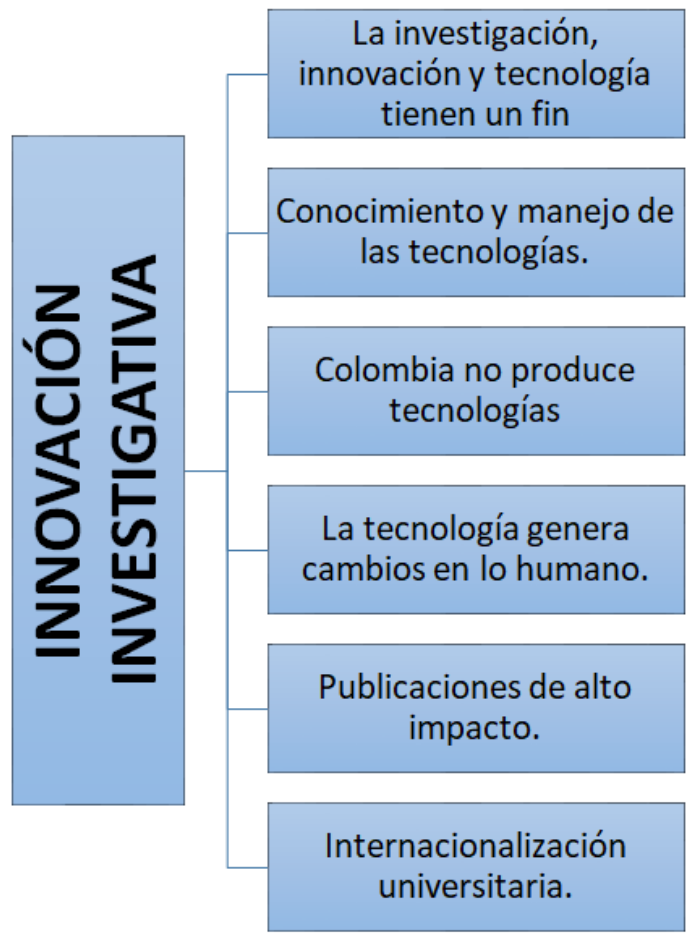

Fuente: elaboración propia.

En lo que sigue no se desarrollará por separado cada uno de estos dos temas que, como se ha dicho, tienen como base dos preguntas hechas a los profesores entrevistados. El trabajo analítico de la información permitió, como se ve en las figuras1 y 2, tematizar en algunas ideas clave lo expresado por los docentes. Por esto, la información que se recoge en estas dos figuras será insumo para problematizar en torno a una tesis emergente en el desarrollo del proyecto de investigación. Esta tesis se enuncia en la siguiente tabla desde una perspectiva de la pedagogía crítica:

Tabla 1. Comparativo del concepto de investigación

\begin{tabular}{l|l}
\multicolumn{1}{c|}{ Tesis emergente } & \multicolumn{1}{c}{ Perspectiva crítica } \\
\hline $\begin{array}{l}\text { 'La emergencia en la época actual de las } \\
\text { universidades que privilegian la investigación } \\
\text { como una función sustantiva ha traspuesto el } \\
\text { sentido y el rol de la docencia hacia una crítica del conocimiento lo } \\
\text { definiría como una construcción social } \\
\text { mercantilización del conocimiento'. }\end{array}$ & $\begin{array}{l}\text { humanas a la intencionalidad y la conducta } \\
\text { conocimiento del aula puede usarse o bien en } \\
\text { provecho de la emancipación o bien en }\end{array}$ \\
& $\begin{array}{l}\text { beneficio de la dominación" (Giroux, 2004, } \\
\text { p. 52). }\end{array}$
\end{tabular}

Fuente: elaboración propia.

Además, se les preguntó a los profesores clasificados en júnior, asociado o sénior: ¿qué herramientas electrónicas TIC, TAC,
TEP o TE usan los docentes para fundamentar los conocimientos impartidos a través de las aulas virtuales de aprendizaje? (tabla 2) 
Tabla 2. Comparativo del uso de herramientas TIC, TAC, TEP, TE en los procesos de investigación y fundamentación en las aulas virtuales de aprendizaje

\begin{tabular}{l|l|}
\multicolumn{1}{c|}{ Tesis emergente } & \multicolumn{1}{c|}{\begin{tabular}{c}
\multicolumn{1}{c|}{ Voces de los docentes } \\
A2: "trabajo TIC, para el aprendizaje y \\
empoderamiento, adicionalmente manejo de \\
tecnologías fáciles de utilizar como Google. \\
Docs, las estadísticas, redes compartidas, \\
foros, trabajo con wiki".
\end{tabular}} \\
$\begin{array}{ll}\text { Los docentes hacen poco uso de las TIC, TAC, } \\
\begin{array}{l}\text { TEP y TE en la fundamentación conceptual de } \\
\text { sus asignaturas en las aulas de aprendizaje } \\
\text { virtual. }\end{array}\end{array}$ & $\begin{array}{l}\text { J2: "total, todo enfocado en las tecnologías } \\
\text { emergentes [...] trabajé una mano robótica, } \\
\text { desarrollé software". }\end{array}$ \\
& $\begin{array}{l}\text { S1: "Utilizamos Moodle y WhatsApp para } \\
\text { compartir conocimiento de la forma más } \\
\text { rápida posible; otro es el uso de las bases de } \\
\text { datos". }\end{array}$ \\
\hline
\end{tabular}

Fuente: elaboración propia.

De otro lado, en el uso de redes de conocimiento en la socialización de los saberes entre los docentes aliados, docentes de la institución y estudiantes se encontró lo expuesto en la tabla 3.

Tabla 3. Comparativo del uso de redes de conocimiento en aulas virtuales de aprendizaje

\begin{tabular}{|c|c|}
\hline Tesis emergente & Voces de los docentes \\
\hline \multirow{3}{*}{$\begin{array}{l}\text { Durante la fundamentación de la } \\
\text { producción científica ha tenido } \\
\text { oportunidad de contar con redes de } \\
\text { conocimiento para su divulgación entre } \\
\text { los estudiantes. }\end{array}$} & $\begin{array}{l}\text { A3: "Se caracterizan por ser temáticas, eso es una } \\
\text { gran ventaja }[\ldots] \text { es decir, un conocimiento colectivo" }\end{array}$ \\
\hline & $\begin{array}{l}\text { J2: "Definitivamente es importantísimo tener un } \\
\text { aliado estratégico y ojalá fuera de la universidad, } \\
\text { fuera de Colombia [...] cómo podemos mezclar esos } \\
\text { dos en conjunto para hacer una sinergia y producir } \\
\text { investigación de punta, de calidad". }\end{array}$ \\
\hline & $\begin{array}{l}\text { S1: "Para que la red sirva: 1) debe haber comunicación } \\
\text { fluida entre las partes; 2) haber el menor número de } \\
\text { intermediarios administrativos para que las cosas se } \\
\text { hagan; y 3) que se generen resultados". }\end{array}$ \\
\hline
\end{tabular}

Fuente: elaboración propia.

\section{Discusión}

Cualificación de un investigador para una universidad de investigación

Escribir acerca de las cualidades de un buen investigador representa, actualmente, una gran responsabilidad. Los profesores entrevistados coinciden generalmente en que un buen perfil de un investigador contiene las siguientes cualidades: interés, motivación, curiosidad, compromiso, conocimiento de un enfoque académico y metodológico. No indica esto que la caracterización y comprensión de estas cualidades sea extremadamente útil para aquellos que comienzan a involucrarse en el campo de la investigación. Más bien tiene que ver con el perfil que puede ir configurándose a través de la práctica investigativa.

Como se ve en la figura 1 , conviene señalar que para ser un buen investigador primero se requiere la intención de participar en la investigación y, en consecuencia, o como un segundo paso, mostrar un interés dedicado a hacer la mejor investigación posible. Del estudio realizado puede inferirse dos cualidades propias de un buen investigador en el campo universitario, a saber:

La primera corresponde a la capacidad analítica y contextual, para la cual el ejercicio de la investigación implica una variedad de factores. Por ejemplo, involucra hacer cuestionamientos y responder a estos, tales como: ¿por qué y para qué quiero hacer esta investigación? ¿Cómo debería desarrollar esta investigación? 
¿Necesitaré de ayuda para investigar? ¿Cuál es la metodología apropiada? ¿Cuál es la pregunta o las preguntas adecuadas para hacer y cómo hacerlas? ¿Dónde y cómo puedo obtener respuestas?, etc. Esta serie de preguntas tienen como base el interés por hacer explícito el sentido y alcance de la investigación como práctica cotidiana de una persona. De igual manera, esta práctica tiene un contexto propio de desarrollo.

La segunda cualidad corresponde a la curiosidad investigativa. Como se indicó, investigar implica de base una intención de hacerlo, pero esta solo tendrá desarrollo si la práctica investigativa se acompaña por una actitud curiosa del investigador. No basta con definir categorías temáticas de trabajo ni un enfoque metodológico claro, ni basta con tener un enfoque o marco temático explícito. Tales herramientas no podrán gestionarse pertinentemente si no hay suficiente curiosidad, puesto que la búsqueda de información y la construcción del saber inquieren profundización, es decir, reclaman curiosidad.

\section{La paradoja de las universidades de investigación}

Como se señaló algunos párrafos atrás, un excelente investigador demanda de al menos dos cualidades claves: el análisis contextual de los fenómenos y la curiosidad para estudiarlos. Empero, estas dos cualidades entran en choque con el sentido actual que se puede encontrar en la investigación como función y práctica universitaria.

En el contexto actual colombiano se ha dado una intensificación y globalización del desarrollo de las universidades de investigación en general. Dado que muy pocas instituciones de educación superior pueden hacer parte de este grupo, se ha configurado para algunos una llamada 'universidad de investigación superior' para enfatizar la perspectiva mundial y la alta producción académica de este 'grupo' de universidades de investigación, que encabezan prácticamente todas las listas de universidades destacadas mundialmente (Baker, 2015).

Diversos autores sugieren que para la clasificación de las universidades de investigación se tienen en cuenta una serie de atributos y características específicas para la práctica investigativa. En este grupo se encuentran, entre otros: Graham y Diamond (2004), Brint (2019), Geiger (2017), Altbach y Balán (2007). Para otros autores estos atributos corresponden o se fundan en la experiencia estadounidense de las últimas décadas, que ha terminado por configurar un modelo de universidad de investigación y que se ha adoptado a nivel mundial (Altbach y Peterson, 2015; Baker, 2015).

Mohrman, Ma y Baker (2008) sugieren que hay una serie de cualidades específicas que definen una universidad de investigación, aun cuando en cada universidad puede variar su desarrollo, a saber: 1) estas universidades ven su misión como una superación transnacional de sus límites geográficos y de las fronteras del conocimiento, es decir, tienen como objetivo educar para una perspectiva global; 2) estas instituciones desarrollan prácticas cada vez más intensivas en investigación con el uso de métodos científicos de diversas disciplinas; 3 ) se promueve el trabajo interinstitucional e internacional en los integrantes de los grupos de investigación, y en sus roles, como productores de nuevos conocimientos, se les pide convertirse en miembros de equipos interdisciplinares e internacionales; 4) existe una diversificación de las fuentes de financiación, las universidades van más allá las contribuciones de los estudiantes o del apoyo del Gobierno, y generan planes para diversificar su base financiera con fondos de corporaciones y donantes privados o externos, que incluyen, por ejemplo, la creación de empresas con fines de lucro como productos derivados la investigación; 5) se crean nuevas relaciones entre universidades, gobiernos y corporaciones para avanzar en el desarrollo económico y producir conocimiento para el bien social; 6) estas universidades adoptan estrategias de 'reclutamiento' en todo el mundo para estudiantes, profesores y administradores; 7) estas instituciones desarrollan procesos de reestructuración permanente interna dirigida a la investigación, que integra directores interdisciplinarios, programas de capacitación tanto de profesores como estudiantes y una mayor infraestructura tecnológica; y 8) se privilegian las alianzas con organizaciones no gubernamentales internacionales y organizaciones multigubernamentales en apoyo a la investigación colaborativa, la movilidad de estudiantes y docentes, y la validación global de los programas académicos.

En ese sentido, la paradoja que acá se cuestiona y que se recoge en la tesis formulada páginas atrás se resume en que, si bien la investigación es una función sustantiva de la universidad actual y de la docencia, ha trastocado la práctica docente universitaria y los fines de la investigación en relación con la academia. En lo que sigue se dará cuenta de los principales asuntos problemáticos que se puede analizar de esta paradoja.

\section{La internacionalización universitaria como uno de los fines de la investigación}

Este tipo de instituciones universitarias se ven en la necesidad de enfatizar y fortalecer la dimensión internacional de su identidad. La arista que más obliga a esto es justamente el hecho de que cada año cientos de miles de estudiantes abandonan sus países de origen y migran a países extranjeros para hacer o continuar sus estudios profesionales, por lo que es necesaria la oferta de estrategias para la retención de los estudiantes.

Desde luego hay otros fenómenos que así lo exigen. Otro de estos es el componente de internacionalización que se les reclama a las universidades y que, en la mayoría de los casos, se mide por el número y el porcentaje de estudiantes extranjeros que llegan a la institución, como también el número y porcentaje de estudiantes y docentes que sale en actividades de intercambio. Para esto, la academia promueve la gestión de acuerdos o convenios formales con otras universidades e institutos de investigación en otros países, lo que a su vez se constituye en otro indicador del alcance internacional de una institución universitaria.

\section{La producción de conocimiento rentable}

Desempeñarse como una universidad de investigación, entre otros propósitos, busca fortalecer la habilidad institucional para producir conocimientos de manera altamente reconocida. Según Geiger (2017), el núcleo de esto es la expansión de las funciones de docencia, investigación y servicio en una institución, que puede describirse mejor como un conglomerado de conocimiento. Desde luego, en este punto es posible cuestionar qué se categoriza o valoriza como nuevo conocimiento al científico y al tecnológico, particularmente el relacionado con las ciencias naturales, por lo que de algún modo la investigación en ciencias humanas y sociales queda relegada y rezagada en la práctica universitaria. De hecho, como denuncian varios autores, en aquellas instituciones en las que se les da alguna cabida, se les exige asimismo asumir enfoques y procesos metodológicos 'verificables' y cuantificables, es decir, seguir el modelo científico tradicional de las ciencias naturales (Abbott, 2010; Powell y Owen\#Smith, 2002, Frank y Meyer, 2019).

En ese sentido, la universidad ha visto la posibilidad de expandirse más allá de ser una institución de educación superior, para configurarse también como una institución con emprendimiento empresarial. Por ejemplo, como sugieren Graham y Diamond (2004), la gran demanda de investigación médica ha vinculado a las facultades de medicina y los laboratorios 
de medicamentos en una relación de investigación altamente expansiva.

En Colombia, por ejemplo, algunas universidades, además de responder a las exigencias del Sistema Nacional de Ciencia, Tecnología e Innovación regentado por Colciencias, han promovido el interés por la producción de conocimiento no solo de alto nivel, sino ampliamente cuantificable, esperando que dichos avances se retribuyan en productos mercantilizables; es decir, que eventualmente reviertan bienes y ganancias a la institución. Como afirman algunos autores, esto ha exigido que en la universidad la investigación vaya más allá de la curiosidad intelectual del investigador o de los grupos de investigación, y proporcione incentivos para que los trabajadores de la ciencia y la tecnología lleven sus desarrollos e innovaciones a la implementación y a la producción de mercado (Fonseca, 2018; Slaughter y Rhoades, 2009).

Al ser la implementación de la investigación un objetivo especial, favorece que las disciplinas más estrechamente afines con el mercado tengan mayor prioridad y relevancia dentro de las universidades de investigación (Chiva y Gil, 2018). Cada vez más, las universidades están creando centros de investigación, oficinas de transferencia de tecnología y, como se indicó atrás, empresas derivadas para llevar sus productos al mercado.

\section{Cambios en la profesión académica y el rol del docente}

El papel y perfil de los docentes se ha modificado también en las universidades, especialmente en aquellas que pretenden destacarse por su función investigativa, pues han exigido a los profesores procesos de capacitación, formación y prácticas más allá de la docencia, por lo que un profesor debe ser un investigador propiamente. Desde luego, es esto un valor ganado para la docencia, empero, esta puede estar subyugada al ejercicio investigativo y desplazar asuntos como la didáctica para una buena enseñanza.

En Colombia, por ejemplo, se ha pasado a categorizar al docente en los escalafones universitarios teniendo como referente los indicadores y la estructura clasificatoria de investigadores en el Ministerio de Ciencia, Tecnología e Innovación (Minciencias). Ya no solo se establece la categoría de profesor auxiliar, asociado o titular, sino que se suman la de investigador reconocido, júnior, asociado o sénior. Como ejemplo, la estabilidad laboral del docente en las instituciones universitarias públicas no la garantiza una buena evaluación de sus estudiantes, como décadas atrás, o sus estudios de posgrado, sino que la determina ampliamente la categoría de investigador que le conceda el Minciencias.

En la universidad de investigación se llega incluso a 'empoderar' a los docentes, vía incentivos u otros medios, para que contribuyan a obtener financiamiento externo, lo que a su vez puede determinar su éxito profesional. Asimismo, ya no basta con publicar resultados de sus investigaciones, sino que estas deben estas especialmente en revistas de habla inglesa o de instituciones extranjeras altamente reconocidas. Como cuestionan Stromquist y colaboradores (2008), las prioridades de las universidades se subyugan a los intereses mercantilistas, llegando incluso a desdibujarse, por lo que la enseñanza, como práctica tradicional, se legitima solo si la universidad deviene como un conglomerado de conocimiento productivo.

De igual forma, los mismos autores sostienen que, como resultado de esto, el ambiente de trabajo académico y el papel de los docentes está cambiando radicalmente, "están ocupados comercializando su conocimiento" (p. 78). Tal es el caso de una nueva categoría de profesores universitarios en muchos países que no ejercen la docencia o no enseñan en absoluto, sino que trabajan en actividades ajenas al aula de clase, por ejemplo, con proyectos licitados y contratados, empresas de consultoría, institutos de investigación y entidades gubernamentales.

Por su parte, Bok (2015) denuncia que la libertad académica se ve seriamente afectada por presiones del mercado, especialmente porque las agencias de financiación - generalmente externas a la universidad - influyen en las prioridades de investigación, por lo que estas libertades pueden verse comprometidas y determinadas. Esto termina configurando la llamada investigación de propiedad y corporativa, que influye tanto en la dirección de la investigación como en el propósito y el medio para compartir abiertamente los resultados. Como consecuencia, los docentes se ven perfilados más como un empleado más de la institución, que como parte fundamental del campus universitario.

De la formación investigativa a la orientación del conocimiento a través del aula virtual

Las universidades de investigación requieren profesionales idóneos al frente de sus grupos y proyectos de investigación, especialmente en la orientación de los aprendizajes de los estudiantes. La fundamentación epistemológica del saber, que cimenta al docente en los procesos metodológicos del conocimiento en su disciplina; el origen, desarrollo, la actualidad y los futuribles de su profesión; la idoneidad en su campo, que garantiza el dominio a profundidad de los saberes, proceso y procedimientos; y, finalmente, la innovación caracterizada por la aplicación y uso pedagógico del saber en la orientación del conocimiento entre sus estudiantes, son los pilares que garantizan a través de aprendizaje de ambientes virtuales un conocimiento fundamental (González, Padilla y Montoya 2019).

\section{Conclusiones}

Afirmar que la emergencia en la época actual de las universidades que privilegian la investigación como una función sustantiva ha traspuesto el sentido y el papel de la docencia hacia una mercantilización del conocimiento, precisa que las universidades en aras de fungir como garantes de la ciencia y la tecnología han asumido la investigación como un camino expedito y mediático para ganar reconocimiento internacional y mercantil, por ejemplo, mediante la gestión de acuerdos o convenios formales con universidades e institutos de investigación en otros países; esto, a su vez, se constituye en otro indicador del alcance internacional de una institución universitaria. Se trata, en últimas, de un aprovechamiento de las diversas oportunidades que surjan para expandir una función universitaria rentable, por lo que la investigación resulta ser la estrategia más oportuna.

Asimismo, se indica que en las universidades de investigación, en particular, los profesores enfrentan una diversidad de exigencias y requerimientos en sus prácticas docentes. Es evidente que incluso los jóvenes profesores, además de acreditar títulos de doctorado, luchan por lograr nombramientos laborales permanentes en el mundo académico, en lugar de vínculos temporales, como son horas cátedra o de medio tiempo. En Colombia - y se estima que en los países en desarrollo- los profesores compiten para obtener plazas docentes, pero deben cumplir una lista amplia de credenciales que años atrás fueron menores.

A esto se suma que en las universidades los profesores tienen múltiples responsabilidades y de diversa índole. Se les exige que realicen investigaciones publicables y a la vez que ejerzan la docencia con estudiantes de pregrado y posgrado, que sus prácticas evidencien proyección social externa, es decir, que presten servicios a sus universidades y que utilicen sus 
conocimientos en beneficio de las comunidades locales. Como se ha indicado, una estrategia asumida para mantener este tipo de roles en un mismo profesor - y evitar la conflictividad - es el sistema de recompensas económicas por las publicaciones desarrolladas, especialmente en revistas prestigiosas.

Contrario a esto, el planteamiento crítico señalado por Giroux (2016) recuerda que, si bien la investigación puede caer en el uso político de control y dominio, su producto mismo, que es la innovación del conocimiento, no puede tener otro fin que la construcción social vinculada al desarrollo humano global.

El fundamento de una educación de calidad a través de los ambientes virtuales de aprendizaje se sustenta en la formación avanzada de los docentes en las universidades o institutos de educación superior. La formación en investigación del docente y el constante uso de tecnologías, como TIC, TAC, TE y TEP, en su trabajo cotidiano genera capacidades, habilidades y competencias que fácilmente se transmiten en su educando a través del ambiente virtual de aprendizaje (González, Padilla y Montoya, 2019).

\section{Referencias}

Abbott, A. (2010). Chaos of Disciplines . University of Chicago Press.

Altbach, P. y Balán, J. (2007). World Class Worldwide: Transforming Research Universities in Asia and Latin America J. H. Press.

Altbach, P. y Peterson, P. (2015). Higher Education as a Projection of America's Soft Power. En Yasushi, W. y McConnell, D., Soft Power Superpowers: Cultura and national assets of Japan and the United States (pp. 37\#53). Routledge.

Araújo, A. (2017). Pedagogia Da Libertação Em Paulo Freire . Paz e Terra.

Baker, D. (2015). Mass Higher Education and the Super Research University. International Higher Education , (49). https://doi. org/10.6017/ihe.2007.49.7991

Bok, D. (2015). Higher education in America: Revised edition . P. U. Press.

Brint, S. (2019). Two cheers for higher education: Why American universities are stronger than ever and how to meet the challenges they face. P. U. Press.

Chiva, O. y Gil, J. (2018). Aprendizaje.servicio universitario: modelos de intervención e investigación en la formación inicial del docente . Ediciones Octaedro, S. L.

Fonseca, C. (2018). Lineamientos y métodos de construcción y evaluación de políticas y estrategias de ciencia, tecnología e innovación diferenciadas regionalmente para el desarrollo territorial sustentable de Colombia [tesis de doctorado, Universidad Pedagógica y Tecnológica de Colombia]. http://re positorio.uptc.edu.co/handle/001/2954

Frank, D. y Meyer, J. (2019). The University and the Global Knowledge Society. P. U. Press.

Geiger, R. (2017). Perspectives on the History of Higher Education (vol. 24). Routledge.

Giroux, H. (2004). Pedagogía y política de la esperanza: teoría, cultura y enseñanza. Amorrortu Editores.

Giroux, H. (2016). Schooling and the Struggle for Public Life: Democracy's Promise and Education's Challenge. Routledge.
González, K., Padilla, E. y Montoya, M. (2019). ..research y tecnologías colaborativas en la educación superior. Editorial Redipe.

Graham, H. y Diamond, N. (2004). The Rise of American Research Universities: Elites and Challengers in the Postwar Era. J. H. Press.

Mohrman, K., Ma, W. y Baker, D. (2008, 5 de marzo). The Research University in Transition: The Emerging Global Model. Higher Education Policy. 21 , 5\#27. https://doi.org/10 .1057/palgrave.hep.8300175

Powell, W. y Owen, J. (2002). The New World of Knowledge Production in the Life Sciences. En P. Altbach y S. U. Press (eds.), The Future of the City of Intellect: The Changing American University (p. 351).

Presidencia de la República de Colombia. (2019, 25 de julio). Decreto 1330. Por el cual se sustituye el Capítulo 2 y se suprime el Capítulo 7 del Título 3 de la Parte 5 del Libro 2 del Decreto 1075 de 2015 - Único Reglamentario del Sector Educación. https://www.funcionpublica.gov.co/eva/gestornor mativo/norma_pdf.php?i=98270

Slaughter, S. y Rhoades, G. (2009). Academic Capitalism and the New Economy: Markets, State, and Higher Education. J. H. Press.

Stromquist, N., Balbachevsky, E., Colatrella, C. y Gil, M. (2008). The Academic Profession in the Globalization Age: Key Trends, Challenges, and Possibilities. En Altbach, P. y Peterson, P, Higher Education in the new century: Global Challenges and Innovative Idea (pp. 1\#33). Sense Publishers.

Notas

* Texto presentado en el IV Foro Internacional de Investigación en Ambientes Virtuales de Aprendizaje 